\title{
Consolidation of a poroelastic half-space with anisotropic permeability and compressible constituents by axisymmetric surface loading
}

\author{
Sarva Jit Singh ${ }^{1, *}$, Raman Kumar ${ }^{2}$ and Sunita Rani ${ }^{2, * *}$ \\ ${ }^{1}$ Department of Mathematics, University of Delhi, South Campus, New Delhi 110 021, India. \\ ${ }^{2}$ Department of Mathematics, Guru Jambheshwar University of Science and Technology, Hisar 125 001, India. \\ *e-mail: s-j_singh@yahoo.com \\ **e-mail: s_b_rani@rediffmail.com
}

The fully coupled Biot quasi-static theory of linear poroelasticity is used to study the consolidation of a poroelastic half-space caused by axisymmetric surface loads. The fluid and solid constituents of the poroelastic medium are compressible and its permeability in the vertical direction is different from its permeability in the horizontal direction. An analytical solution of the governing equations is obtained by taking the displacements and the pore pressure as the basic state variables and using a combination of the Laplace and Hankel transforms. The problem of an axisymmetric normal load is discussed in detail. An explicit analytical solution is obtained for normal disc loading. Detailed numerical computations reveal that the anisotropy in permeability as well as the compressibilities of the fluid and solid constituents of the poroelastic medium have significant effects on the consolidation of the half-space. The anisotropy in permeability may accelerate the consolidation process and may lead to a dilution in the theoretical prediction of the Mandel-Cryer effect. The compressibility of the solid constituents may also accelerate the consolidation process.

\section{Introduction}

Biot's theory of linear poroelasticity (Biot 1941, 1956) has been used very extensively to study the consolidation of a homogeneous or layered half-space by surface loads or buried sources (see, e.g., Rudnicki 1986; Pan 1999; Wang and Kuempel 2003; Singh and Rani 2006). However, most of these studies did not take into account the anisotropy in hydraulic permeability. Permeability determines the ability of the porous medium to conduct fluid flow in its pores and, therefore, can be different in different directions. In most cases, the soil deposits are the result of a sedimentation process which produces horizontal stratification. Consequently, permeabilities in the horizontal and vertical directions may differ. It has been found experimentally that the horizontal permeability of soil may be an order of magnitude, or more, greater than the vertical permeability. This degree of hydraulic anisotropy will have significant effect on the consolidation process. As mentioned by Ganbe and Kurashige (2001), the permeability is anisotropic in many oil/gas reservoirs. In some structures, the permeability anisotropy in the horizontal direction to the vertical direction is as much as 100:1. Anisotropy from $2: 1$ to $3: 1$ is common, especially in naturally fissured media. It is, therefore, useful to study the effect of the anisotropy in permeability on the consolidation of a poroelastic half-space under an externally applied load due to its possible geophysical or engineering applications.

Booker and Randolph (1984) discussed the consolidation of a soil medium with transversely isotropic deformation and flow properties due to surface loading. Both the pore water and the soil

Keywords. Anisotropic permeability; compressible constituents; poroelastic half-space; quasi-static deformation; surface loading. 
grains were assumed to be incompressible. Booker and Carter (1986) obtained a closed-form solution for the long-term deformation and pore pressure in a poroelastic half-space caused by withdrawal of pore fluid at a constant rate from a point sink. The soil was assumed to be transversely isotropic with respect to its flow properties. Booker and Carter (1987a) presented a solution for the transient effects of pumping fluid from a point sink embedded in a poroelastic half-space possessing anisotropic permeability and incompressible fluid and solid constituents. In a subsequent study, Booker and Carter (1987b) obtained the corresponding solution for a poroelastic half-space with compressible fluid but incompressible solid constituents.

Chen (2004) studied the consolidation of a multilayered poroelastic half-space with anisotropic permeability and compressible fluid constituents. The solid constituents were assumed to be incompressible. For such a poroelastic material, the BiotWillis coefficient $\alpha=1$. In a subsequent study, Chen (2005) discussed the steady-state response of multilayered poroelastic half-space to a point sink. Both the permeability and the poroelasticity of the medium were assumed to be transversely isotropic, but its fluid and solid constituents were assumed to be incompressible. In a recent study, Singh et al (2007) discussed the quasi-static plane strain deformation of a poroelastic half-space with anisotropic permeability and compressible constituents by twodimensional surface loads. An analytical solution was obtained by using a pure compliance formulation in which the stresses and the pore pressure are taken as the basic state variables. Biot's stress function was used to decouple the governing equations. The problem of normal strip loading was discussed in detail.

The purpose of the present paper is to study the consolidation of a poroelastic half-space by an axisymmetric surface loading. The permeability in the vertical direction may be different from the permeability in the horizontal direction. The fluid and solid constituents of the poroelastic medium are compressible. A stiffness formulation is used, taking the displacements and the pore pressure as the basic state variables. An analytical solution of the governing equations is obtained by taking the Laplace transform with respect to time and the Hankel transform with respect to the space variables. The problem of normal disc loading is discussed in detail. An explicit analytical solution in the Laplace-Hankel transforms domain is obtained. Detailed numerical computations are performed to study the effects of the anisotropy in permeability and the compressibilities of the fluid and solid constituents.

\section{Basic equations}

Let $(r, \theta, z)$ denote the cylindrical polar coordinates and $\left(u_{r}, u_{\theta}, u_{z}\right)$ the corresponding displacement components. For axial symmetry, $\partial / \partial \theta \equiv 0$, and the strain components are:

$$
\begin{aligned}
& \epsilon_{r r}=\frac{\partial u_{r}}{\partial r}, \quad \epsilon_{\theta \theta}=\frac{u_{r}}{r}, \quad \epsilon_{z z}=\frac{\partial u_{z}}{\partial z} \\
& \epsilon_{r z}=\frac{1}{2}\left(\frac{\partial u_{r}}{\partial z}+\frac{\partial u_{z}}{\partial r}\right), \quad \epsilon_{r \theta}=\epsilon_{\theta z}=0 .
\end{aligned}
$$

A homogenous elastically isotropic poroelastic material with compressible fluid and solid constituents can be characterized by four constitutive constants. Let these constants be: the shear modulus $(G)$, the drained Poisson's ratio $(\nu)$, the undrained Poisson's ratio $\left(\nu_{u}\right)$ and the Biot-Willis coefficient $(\alpha)$. In terms of the bulk moduli, $\alpha$ and $\nu_{u}$ can be expressed in the form:

$$
\begin{aligned}
\alpha & =1-\frac{K}{K_{s}} \quad(0 \leq \alpha \leq 1), \\
\nu_{u} & =\frac{3 K_{u}-2 G}{2\left(3 K_{u}+G\right)} \quad\left(0 \leq \nu \leq \nu_{u} \leq \frac{1}{2}\right),
\end{aligned}
$$

where $K$ is the drained bulk modulus, $K_{u}$ is the undrained bulk modulus and $K_{s}$ is the bulk modulus of the solid phase of the poroelastic material. For axial symmetry the components of the total stress tensor $(\overrightarrow{\vec{\sigma}})$, the total strain tensor $(\overrightarrow{\vec{\epsilon}})$, and the pore pressure $(p)$ are related by the following system of equations (Detournay and Cheng 1993; Wang 2000):

\subsection{Equilibrium equations}

$$
\begin{aligned}
& \frac{\partial^{2} u_{r}}{\partial r^{2}}+\frac{1}{r} \frac{\partial u_{r}}{\partial r}-\frac{u_{r}}{r^{2}}+\frac{\partial^{2} u_{r}}{\partial z^{2}} \\
& +\frac{1}{1-2 \nu} \frac{\partial \epsilon}{\partial r}-\frac{\alpha}{G} \frac{\partial p}{\partial r}=0 \\
& \frac{\partial^{2} u_{z}}{\partial r^{2}}+\frac{1}{r} \frac{\partial u_{z}}{\partial r}+\frac{\partial^{2} u_{z}}{\partial z^{2}}+\frac{1}{1-2 \nu} \frac{\partial \epsilon}{\partial z}-\frac{\alpha}{G} \frac{\partial p}{\partial z}=0
\end{aligned}
$$

where

$$
\epsilon=\operatorname{div} \mathbf{u}=\frac{\partial u_{r}}{\partial r}+\frac{u_{r}}{r}+\frac{\partial u_{z}}{\partial z}
$$


denotes the dilatation. From equations (2) to (4), we obtain

$$
\eta \nabla^{2} p=G \nabla^{2} \epsilon
$$

where

$$
\eta=\frac{1-2 \nu}{2(1-\nu)} \alpha
$$

is the poroelastic stress coefficient.

\subsection{Constitutive equations}

$$
\sigma_{i j}=2 G\left(\epsilon_{i j}+\frac{\nu}{1-2 \nu} \epsilon \delta_{i j}\right)-\alpha p \delta_{i j}
$$

where the subscripts $i$ and $j$ can be $r, \theta$ or $z$.

\subsection{Darcy's law}

According to Darcy's law of fluid flow in a poroelastic medium with anisotropic permeability

$q_{r}=-\chi_{r} \partial p / \partial r, \quad q_{\theta}=0, \quad q_{z}=-\chi_{z} \partial p / \partial z$

where $\mathbf{q}$ is the fluid flux and $\left(\chi_{r}, \chi_{z}\right)$ is the Darcy conductivity in the $(r, z)$ direction.

\subsection{Continuity equation}

$$
\operatorname{div} \mathbf{q}=-\frac{\partial}{\partial t}\left(\alpha \epsilon+\frac{1}{M} p\right)
$$

where

$$
M=\frac{2 G\left(\nu_{u}-\nu\right)}{\alpha^{2}(1-2 \nu)\left(1-2 \nu_{u}\right)},
$$

is the Biot modulus.

\subsection{Fluid diffusion equation}

Combining the continuity equation and Darcy's law, we obtain the fluid diffusion equation in the form

$$
\chi_{r}\left(\frac{\partial^{2} p}{\partial r^{2}}+\frac{1}{r} \frac{\partial p}{\partial r}\right)+\chi_{z} \frac{\partial^{2} p}{\partial z^{2}}=\frac{\partial}{\partial t}\left(\alpha \epsilon+\frac{1}{M} p\right)
$$

This is a coupled diffusion equation in pore pressure for a poroelastic material possessing anisotropic permeability and compressible fluid and solid constituents. If we put $\alpha=1$ in equations (7) and (11), we get the corresponding equations of Chen (2004) for axial symmetry. Since $\alpha=1$ for incompressible solid constituents, the results of Chen (2004) are valid for the particular case of a poroelastic material with incompressible solid constituents.

\section{Solution of the governing equations}

\subsection{Pore pressure}

Eliminating $\epsilon$ from equations (5) and (11), we obtain:

$$
\left[c_{r}\left(\frac{\partial^{2}}{\partial r^{2}}+\frac{1}{r} \frac{\partial}{\partial r}\right)+c_{z} \frac{\partial^{2}}{\partial z^{2}}-\frac{\partial}{\partial t}\right] \nabla^{2} p=0
$$

where

$$
\left(c_{r}, c_{z}\right)=\frac{2 G(1-\nu)\left(\nu_{u}-\nu\right)}{\alpha^{2}(1-2 \nu)^{2}\left(1-\nu_{u}\right)}\left(\chi_{r}, \chi_{z}\right)
$$

is the hydraulic diffusivity. Taking the Laplace transform of equation (12), we have

$$
\left[c_{r}\left(\frac{\partial^{2}}{\partial r^{2}}+\frac{1}{r} \frac{\partial}{\partial r}\right)+c_{z} \frac{\partial^{2}}{\partial z^{2}}-s\right] \nabla^{2} \tilde{p}=0
$$

where

$$
\tilde{p}(r, z, s)=\int_{0}^{\infty} p(r, z, t) e^{-s t} d t
$$

is the Laplace transform of $p(r, z, t)$.

We define the $n$th order Hankel transform $\bar{f}_{n}(k, z, s)$ of a function $\tilde{f}(r, z, s)$ by the relation

$$
\bar{f}_{n}(k, z, s)=\int_{0}^{\infty} \tilde{f}(r, z, s) J_{n}(k r) r d r
$$

so that

$$
\tilde{f}(r, z, s)=\int_{0}^{\infty} \bar{f}_{n}(k, z, s) J_{n}(k r) k d k
$$


Taking the zeroth-order Hankel transform of equation (14), we obtain

$$
\left(\frac{d^{2}}{d z^{2}}-m^{2}\right)\left(\frac{d^{2}}{d z^{2}}-k^{2}\right) \bar{p}_{0}(k, z, s)=0
$$

where

$$
m^{2}=\left(\frac{c_{r}}{c_{z}}\right) k^{2}+\frac{s}{c_{z}} .
$$

Suitable solutions of equation (18) which are bounded for $z \rightarrow \infty$ are of the form

$$
\bar{p}_{0}=A_{1} e^{-m z}+A_{2} e^{-k z},
$$

where the arbitrary constants $A_{1}, A_{2}$ may be functions of $s$ and $k$.

\subsection{Dilatation}

Equations (5) and (20) yield

$$
G\left(\frac{d^{2}}{d z^{2}}-k^{2}\right) \bar{\epsilon}_{0}=\eta\left(m^{2}-k^{2}\right) A_{1} e^{-m z}
$$

Solving, we have

$$
\bar{\epsilon}_{0}(k, z, s)=\left(\frac{\eta}{G}\right) A_{1} e^{-m z}+A_{3} e^{-k z} .
$$

Inserting the expressions for $\bar{p}_{0}$ and $\bar{\epsilon}_{0}$ from equations (20) and (21) into the Laplace-Hankel transformed equation (11), we have

$$
\begin{aligned}
\frac{A_{3}}{A_{2}}= & \frac{\alpha(1-2 \nu)\left(1-2 \nu_{u}\right)}{2 G\left(\nu_{u}-\nu\right)} \\
& \times\left[\left(c_{z}-c_{r}\right) \frac{(1-2 \nu)\left(1-\nu_{u}\right)}{(1-\nu)\left(1-2 \nu_{u}\right)} \frac{k^{2}}{s}-1\right] .
\end{aligned}
$$

\subsection{Displacements}

Taking the Laplace transform followed by the first order Hankel transform of equation (2), using equation (20) and solving the resulting ordinary differential equation of the second order we obtain:

$$
\begin{aligned}
\bar{u}_{r, 1}(k, z, s)= & \int_{0}^{\infty} \tilde{u}_{r}(r, z, s) J_{1}(k r) r d r \\
= & -\frac{\eta k}{G\left(m^{2}-k^{2}\right)} A_{1} e^{-m z} \\
& +\frac{1}{2}\left(\frac{\alpha}{G} A_{2}-\frac{A_{3}}{1-2 \nu}\right) z e^{-k z}+A_{4} e^{-k z},
\end{aligned}
$$

where $A_{4}$ is an arbitrary constant. Similarly, equation (3) yields

$$
\begin{aligned}
\bar{u}_{z, 0}= & \frac{-\eta m}{G\left(m^{2}-k^{2}\right)} A_{1} e^{-m z} \\
& +\frac{1}{2}\left(\frac{\alpha}{G} A_{2}-\frac{A_{3}}{1-2 \nu}\right) z e^{-k z}+A_{5} e^{-k z} .
\end{aligned}
$$

Equation (4) implies

$$
\bar{\epsilon}_{0}=k \bar{u}_{r, 1}+\frac{d}{d z} \bar{u}_{z, 0}
$$

From equations (21), (23) and (24), we find

$$
k\left(A_{4}-A_{5}\right)=-\frac{\alpha}{2 G} A_{2}+\frac{3-4 \nu}{2(1-2 \nu)} A_{3} .
$$

\subsection{Stresses}

Equations (1), (7), (20), and (21) yield

$$
\begin{aligned}
\frac{\bar{\sigma}_{r z, 1}}{2 G}= & \frac{\eta m k}{G\left(m^{2}-k^{2}\right)} A_{1} e^{-m z} \\
& +\frac{1}{4}\left(\frac{\alpha}{G} A_{2}-\frac{A_{3}}{1-2 \nu}\right)(1-2 k z) e^{-k z} \\
& -\frac{1}{2} k\left(A_{4}+A_{5}\right) e^{-k z}
\end{aligned}
$$




$$
\begin{aligned}
\frac{\bar{\sigma}_{z z, 0}}{2 G}= & \frac{\eta k^{2}}{G\left(m^{2}-k^{2}\right)} A_{1} e^{-m z} \\
& -\frac{1}{2}\left(\frac{\alpha}{G} A_{2}-\frac{A_{3}}{1-2 \nu}\right)(1+k z) e^{-k z} \\
& +\left(\frac{1}{2} A_{3}-k A_{4}\right) e^{-k z}
\end{aligned}
$$

$$
\begin{aligned}
\tilde{\sigma}_{z z}(r, z, s)= & 2 G \int_{0}^{\infty}\left[\frac{\eta k^{2}}{G\left(m^{2}-k^{2}\right)} A_{1} e^{-m z}\right. \\
& -A_{6}(1+k z) e^{-k z} \\
& \left.+\frac{1}{2}\left(A_{3}-2 k A_{4}\right) e^{-k z}\right] J_{0}(k r) k d k,
\end{aligned}
$$

Using equations (17), (22) and (26), the Laplace transform of the solution can be expressed in the form

$$
\begin{gathered}
\tilde{p}(r, z, s)=\int_{0}^{\infty}\left(A_{1} e^{-m z}+A_{2} e^{-k z}\right) J_{0}(k r) k d k, \\
\tilde{q}_{z}(r, z, s)=\chi_{z} \int_{0}^{\infty}\left(m A_{1} e^{-m z}+k A_{2} e^{-k z}\right) J_{0}(k r) k d k,
\end{gathered}
$$

$$
\tilde{\epsilon}(r, z, s)=\int_{0}^{\infty}\left(\frac{\eta}{G} A_{1} e^{-m z}+A_{3} e^{-k z}\right) J_{0}(k r) k d k,
$$

$$
\begin{aligned}
\tilde{u}_{r}(r, z, s)= & \int_{0}^{\infty}\left[\frac{-\eta k}{G\left(m^{2}-k^{2}\right)} A_{1} e^{-m z}\right. \\
& \left.+A_{6} z e^{-k z}+A_{4} e^{-k z}\right] J_{1}(k r) k d k,
\end{aligned}
$$

$$
\begin{aligned}
\tilde{u}_{z}(r, z, s)= & \int_{0}^{\infty}\left[\frac{-\eta m}{G\left(m^{2}-k^{2}\right)} A_{1} e^{-m z}\right. \\
& \left.+A_{6} z e^{-k z}+A_{5} e^{-k z}\right] J_{0}(k r) k d k,
\end{aligned}
$$

$$
\begin{aligned}
\tilde{\sigma}_{r z}(r, z, s)= & 2 G \int_{0}^{\infty}\left[\frac{\eta m k}{G\left(m^{2}-k^{2}\right)} A_{1} e^{-m z}\right. \\
& \left.-A_{6} k z e^{-k z}+\frac{1}{2}\left(A_{3}-2 k A_{4}\right) e^{-k z}\right] \\
& \times J_{1}(k r) k d k
\end{aligned}
$$

$A_{3}=\frac{\eta}{G}\left[1-(1-2 \nu) \frac{\left(1-\nu_{u}\right)}{\left(\nu_{u}-\nu\right)} \frac{s_{a}}{s}\right] A_{2}$

$A_{5}=A_{4}+\frac{\eta}{2 G k}\left[-1+(3-4 \nu) \frac{\left(1-\nu_{u}\right) s_{a}}{\left(\nu_{u}-\nu\right) s}\right] A_{2}$,

$$
\begin{aligned}
A_{6} & =\frac{1}{2}\left(\frac{\alpha}{G} A_{2}-\frac{A_{3}}{1-2 \nu}\right)=\frac{\eta}{2 G}\left[1+\frac{\left(1-\nu_{u}\right) s_{a}}{\left(\nu_{u}-\nu\right) s}\right] \begin{array}{c}
A_{2}, \\
s_{a}
\end{array}=s+\left(c_{r}-c_{z}\right) k^{2}=c_{z}\left(m^{2}-k^{2}\right) .
\end{aligned}
$$

Three arbitrary constants, $A_{1}, A_{2}$ and $A_{4}$, appear in the solution given by equations (29) to (35). These constants have to be determined from the boundary conditions.

\section{Isotropic permeability}

For isotropic permeability, $\chi_{r}=\chi_{z}=\chi, c_{r}=c_{z}=$ $c, s_{a}=s$ and the solution is given by equations (29) to (35) with

$$
\begin{aligned}
& A_{3}=-\frac{\eta(1-\nu)\left(1-2 \nu_{u}\right)}{G\left(\nu_{u}-\nu\right)} A_{2}, \\
& A_{5}=A_{4}+\frac{\eta(1-\nu)\left(3-4 \nu_{u}\right)}{2 G k\left(\nu_{u}-\nu\right)} A_{2}, \\
& A_{6}=\frac{\eta(1-\nu)}{2 G\left(\nu_{u}-\nu\right)} A_{2}, \\
& m^{2}=k^{2}+\frac{s}{c}
\end{aligned}
$$


We have verified that the poroelastic solution for isotropic permeability obtained by us as a particular case of the general solution for anisotropic permeability coincides with the solution given by Wang $(2000)^{*}$.

\section{Surface loading}

Consider a uniform poroelastic half-space $z \geq 0$ with $z$-axis drawn vertically downwards, into the medium. For prescribed surface loads, boundary conditions are of the form

$$
\sigma_{r z}=\left(\sigma_{r z}\right)_{0}, \quad \sigma_{z z}=\left(\sigma_{z z}\right)_{0}, \quad p=0,
$$

at $z=0$, assuming permeable surface. Let

$$
\begin{aligned}
\left(\sigma_{r z}\right)_{0} & =\int_{0}^{\infty} S_{0}(k, s) J_{1}(k r) k d k, \\
\left(\sigma_{z z}\right)_{0} & =\int_{0}^{\infty} N_{0}(k, s) J_{0}(k r) k d k .
\end{aligned}
$$

Equations (29) and (34)-(38) yield

$$
\begin{aligned}
A_{1}= & -A_{2}=\left(\nu-\nu_{u}\right)(m+k)\left(N_{0}-S_{0}\right) \frac{s}{\eta \Omega}, \\
A_{3}= & {\left[s\left(\nu_{u}-\nu\right)-s_{a}\left(1-\nu_{u}\right)(1-2 \nu)\right] } \\
& \times\left(N_{0}-S_{0}\right) \frac{m+k}{G \Omega}, \\
A_{4}= & -\frac{S_{0}}{2 G k}+\left[s\left(\nu_{u}-\nu\right)\left(k^{2}-m^{2}+2 m k\right)\right. \\
& \left.+s_{a}\left(1-\nu_{u}\right)(1-2 \nu)\left(m^{2}-k^{2}\right)\right] \\
& \times \frac{\left(S_{0}-N_{0}\right)}{2 G k(m-k) \Omega}, \\
A_{5}= & -\frac{S_{0}}{2 G k}+\left[s\left(\nu_{u}-\nu\right) m k\right. \\
& \left.-s_{a}(1-\nu)\left(1-\nu_{u}\right)\left(m^{2}-k^{2}\right)\right] \frac{\left(S_{0}-N_{0}\right)}{G k(m-k) \Omega},
\end{aligned}
$$

${ }^{*}$ The expression for $\tilde{\sigma}_{z r}$ given in equation (9.48) of Wang (2000) is incorrect. The correct expression is obtained by replacing $C_{0}$ and $D_{0}$ by $\frac{1}{2} C_{0}$ and $\frac{1}{2} D_{0}$, respectively, in equation (9.48) of Wang (2000).

$$
A_{6}=\left[s\left(\nu_{u}-\nu\right)+s_{a}\left(1-\nu_{u}\right)\right]\left(N_{0}-S_{0}\right) \frac{m+k}{2 G \Omega},
$$

where

$$
\Omega=s\left(\nu_{u}-\nu\right)(k-m)-s_{a}\left(1-\nu_{u}\right)(k+m) .
$$

\subsection{Normal disc loading}

Suppose a total normal force $Q_{0}$ is uniformly applied over a circular surface area $(z=0, r \leq a)$ of radius $a$ with its centre at the origin. If the surface is permeable and the load is applied in the positive $z$-direction, the boundary conditions yield

$$
\begin{gathered}
p=0, \quad \sigma_{r z}=0, \\
\sigma_{z z}= \begin{cases}-\frac{Q_{0}}{\pi a^{2}}, & r \leq a \\
0, & r>a\end{cases}
\end{gathered}
$$

at $z=0$. This implies

$$
S_{0}=0, \quad N_{0}=-\frac{Q_{0}}{\pi} \frac{J_{1}(a k)}{a k} .
$$

Equations (29) to (39) yield the solution in the transform domain:

$$
\begin{aligned}
\tilde{p}(r, z, s)= & \frac{\left(\nu_{u}-\nu\right) s Q_{0}}{\pi a \eta} \int_{0}^{\infty}\left(e^{-m z}-e^{-k z}\right) J_{0}(k r) \\
& \times J_{1}(k a) \frac{(m+k)}{\Omega} d k, \\
\tilde{q}_{z}(r, z, s)= & \frac{\left(\nu_{u}-\nu\right) \chi_{z} s Q_{0}}{\pi a \eta} \int_{0}^{\infty}\left(m e^{-m z}-k e^{-k z}\right) \\
& \times J_{0}(k r) J_{1}(k a) \frac{(m+k)}{\Omega} d k, \\
\tilde{\epsilon}(r, z, s)= & \frac{Q_{0}}{\pi a G} \int_{0}^{\infty}\left[\left(\nu_{u}-\nu\right) s e^{-m z}-\left\{s\left(\nu_{u}-\nu\right)\right.\right. \\
& \left.\left.-s_{a}(1-2 \nu)\left(1-\nu_{u}\right)\right\} e^{-k z}\right] \\
& \times J_{0}(k r) J_{1}(k a) \frac{(m+k)}{\Omega} d k,
\end{aligned}
$$




$$
\begin{aligned}
& \tilde{u}_{r}(r, z, s)=\frac{Q_{0}}{\pi a G} \int_{0}^{\infty}\left[\frac{\left(\nu_{u}-\nu\right) s}{(k-m)}\left(k e^{-m z}-m e^{-k z}\right)\right. \\
& -\frac{1}{2}\left\{s\left(\nu_{u}-\nu\right)+s_{a}\left(1-\nu_{u}\right)\right\}(m+k) z e^{-k z} \\
& -\frac{1}{2}\left\{s\left(\nu_{u}-\nu\right)-s_{a}(1-2 \nu)\right. \\
& \left.\left.\quad \times\left(1-\nu_{u}\right)\right\} \frac{(m+k)}{k} e^{-k z}\right] \\
& \quad \times J_{1}(k r) J_{1}(k a) \frac{1}{\Omega} d k,
\end{aligned}
$$$$
\tilde{u}_{z}(r, z, s)=\frac{Q_{0}}{\pi a G} \int_{0}^{\infty}\left[\frac{\left(\nu_{u}-\nu\right) m s}{k-m}\left(e^{-m z}-e^{-k z}\right)\right.
$$$$
-\frac{1}{2}\left\{s\left(\nu_{u}-\nu\right)+s_{a}\left(1-\nu_{u}\right)\right\}(m+k) z e^{-k z}
$$$$
\left.-(1-\nu)\left(1-\nu_{u}\right) s_{a}\left(\frac{m+k}{k}\right) e^{-k z}\right]
$$$$
\times J_{0}(k r) J_{1}(k a) \frac{1}{\Omega} d k
$$$$
\tilde{\sigma}_{r z}(r, z, s)=\frac{Q_{0}}{\pi a} \int_{0}^{\infty}\left[\frac{2\left(\nu_{u}-\nu\right) m s}{m-k}\left(e^{-m z}-e^{-k z}\right)\right.
$$$$
+\left\{s\left(\nu_{u}-\nu\right)+s_{a}\left(1-\nu_{u}\right)\right\}
$$$$
\left.\times(m+k) z e^{-k z}\right] J_{1}(k r) J_{1}(k a) \frac{k}{\Omega} d k,
$$$$
\tilde{\sigma}_{z z}(r, z, s)=\frac{Q_{0}}{\pi a} \int_{0}^{\infty}\left[\frac{2\left(\nu_{u}-\nu\right) k s}{m-k}\left(k e^{-m z}-m e^{-k z}\right)\right.
$$$$
+\left\{s\left(\nu_{u}-\nu\right)+s_{a}\left(1-\nu_{u}\right)\right\}(m+k)
$$$$
\left.\times(1+k z) e^{-k z}\right] J_{0}(k r) J_{1}(k a) \frac{1}{\Omega} d k .
$$

The results for a concentrated normal load $Q_{0}$ can be obtained from equations (48) to (54) by using the limit

$$
\lim _{a \rightarrow 0} \frac{J_{1}(a k)}{a k}=\frac{1}{2}
$$

\section{Numerical results and discussion}

Equations (48) to (54) constitute a solution of the problem of consolidation of a poroelastic half-space with anisotropic permeability and compressible fluid and solid constituents. The consolidation is caused by a normal disc load $Q_{0}$ distributed uniformly over the circular region $r \leq a$ of the permeable surface $z=0$ of the half-space. The solution obtained is in the Laplace-Hankel transforms domain. Two integrations are required to be performed to get the solution in the physical domain. Schapery (1962) proposed a very simple and efficient approximate formula for finding the Laplace inversion numerically. According to this formula

$$
\phi(t) \approx[s \tilde{\phi}(s)]_{s=1 /(2 t)},
$$

where $\tilde{\phi}(s)$ is the Laplace transform of $\phi(t)$. Accuracy of this formula has been demonstrated, amongst others, by Rajapakse and Senjuntichai (1993), Senjuntichai and Rajapakse (1995) and Chau (1996). In view of its computational efficiency and simplicity, we have used Schapery's approximate formula for the Laplace inversion. The Hankel transform inversion has been performed numerically by using the extended Simpson's rule.

We have computed the surface subsidence $u_{z}$ (also known as settlement) at the centre of the normal disc load and pore pressure at various points on the z-axis (central line). We define the following dimensionless quantities

$$
\begin{aligned}
& P=\left(\frac{\pi a^{2}}{Q_{0}}\right) p, \quad W=\left(\frac{\pi a G}{Q_{0}}\right) u_{z}, \\
& Z=\frac{z}{a}, \quad T=\left(\frac{G \chi_{z}}{a^{2}}\right) t, \quad \gamma^{2}=\frac{c_{r}}{c_{z}}=\frac{\chi_{r}}{\chi_{z}} .
\end{aligned}
$$

Therefore, $W$ denotes the dimensionless subsidence, $P$ the dimensionless pore pressure, $T$ the dimensionless time, $Z$ the dimensionless depth below the surface of the half-space and $\gamma^{2}$ the ratio of the horizontal permeability $\chi_{r}$ to the vertical permeability $\chi_{z}$. 


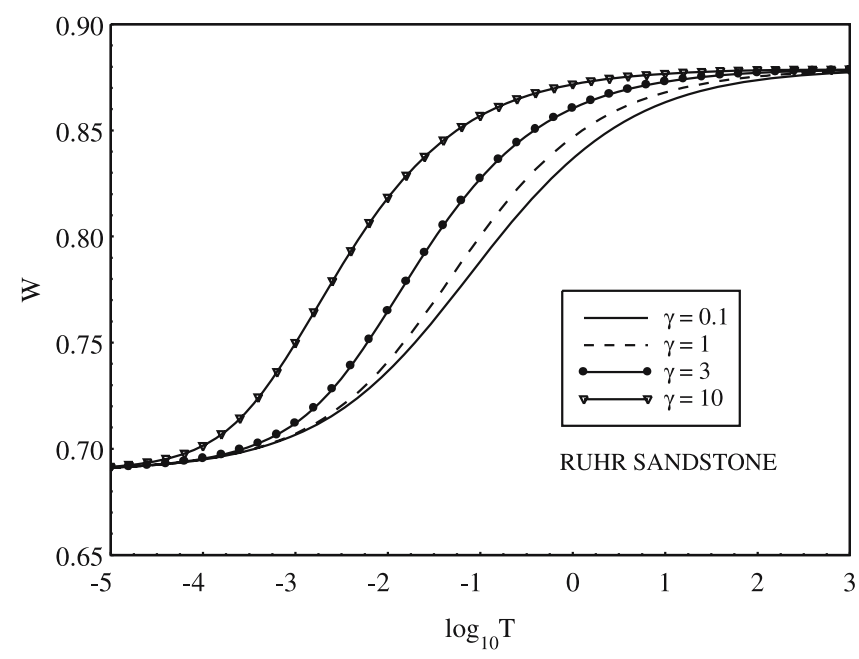

Figure 1. Effect of the permeability anisotropy on the time-settlement. $W$ is the dimensionless displacement at the origin $(r=z=0)$ in the vertical (down) direction, $T$ is the dimensionless time and $\gamma=\left(\chi_{r} / \chi_{z}\right)^{1 / 2}$ is the permeability anisotropy parameter. $\gamma=1$ for a medium with isotropic permeability. For $\gamma>1$, the anisotropy in permeability accelerates the consolidation process.

Figure 1 shows the time-history of the dimensionless subsidence $W$ at the centre of the disc load $(r=z=0)$ for four values of the permeability anisotropy parameter $\gamma$ for Ruhr sandstone for which $\nu=0.12, \nu_{u}=0.31$ and $\alpha=0.65$ (Detournay and Cheng 1993; table 4). When $\gamma=1$, the vertical permeability is equal to the horizontal permeability. We notice that the permeability anisotropy has no effect on the initial settlement or the final settlement. However, if the horizontal permeability $\chi_{r}$ is greater than the vertical permeability $\chi_{z}$ (i.e., if $\gamma>1$ ), the permeability anisotropy accelerates the consolidation process.

Figure 2 depicts the effect of the value of the Biot-Willis coefficient $\alpha$ on the time-history of the dimensionless subsidence $W$ for $\gamma=1, \nu=0.25$ and $\nu_{u}=0.27$. For a poroelastic material with incompressible solid constituents, $\alpha=1$. As $\alpha$ decreases, the compressibility of the solid constituents increases. Figure 2 shows that the compressibility of the solid constituents accelerates the consolidation process.

The influence of the value of the undrained Poisson's ratio $\nu_{u}$ on the time-history of the dimensionless subsidence $W$ is displayed in figure 3 for $\gamma=1, \nu=0.12$ and $\alpha=0.65$. It is known that $\nu \leq \nu_{u} \leq 0.5$. The upper limit $\nu_{u}=0.5$ corresponds to a poroelastic material with incompressible fluid constituents. As expected, the final settlement is independent of the value of $\nu_{u}$. However, the compressibility of the fluid constituents of the poroelastic medium has a strong influence on the consolidation process. The initial settlement for a compressible fluid constituents model is greater

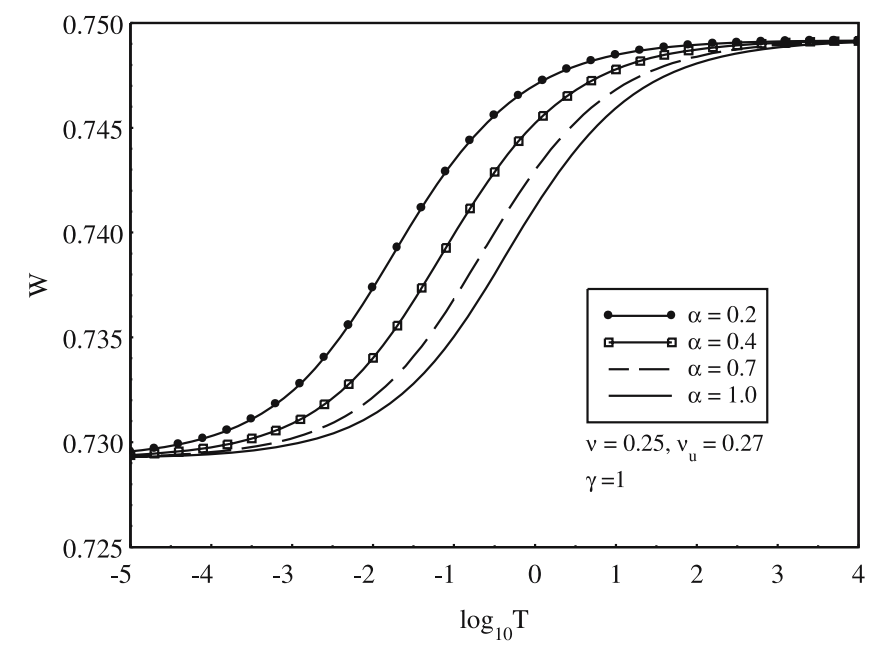

Figure 2. Effect of the compressibility of the solid constituents on the time-settlement for $\nu=0.25, \nu_{u}=0.27$, $\gamma=1$. For a poroelastic model with incompressible solid constituents, $\alpha=1$. The compressibility of the solid constituents accelerates the consolidation process.

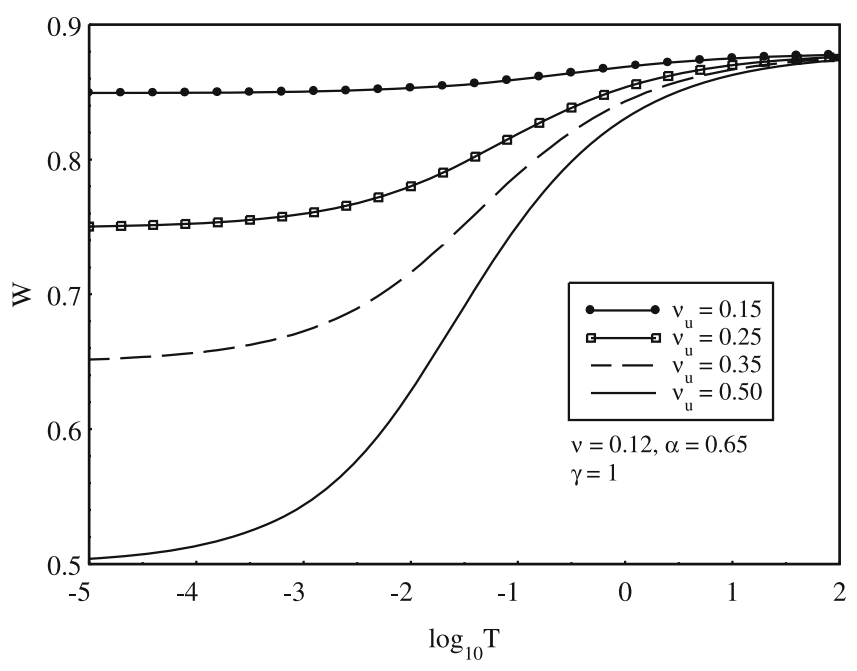

Figure 3. Effect of the compressibility of the fluid constituents on the time-settlement for $\nu=0.12, \alpha=0.65$, $\gamma=1$. For a poroelastic model with incompressible fluid constituents, $\nu_{u}=0.5$. The influence of the compressibility of the fluid constituents is to increase the initial settlement. The final settlement is not affected.

than the initial settlement for the corresponding incompressible fluid constituents model.

Figure 4 shows the effect of the permeability anisotropy on the diffusion of the pore pressure with time. The pore pressure vanishes in the drained state $(T \rightarrow \infty)$. Moreover, anisotropy has no effect in the undrained state $(T \rightarrow 0)$. From figure 4 , we notice that instead of decreasing monotonically with time, the pore pressure rises above the initial undrained value before it decays to zero as $T \rightarrow \infty$. This is in accordance with the Mandal-Cryer effect (Cryer 1963; Detournay and Cheng 1993). 

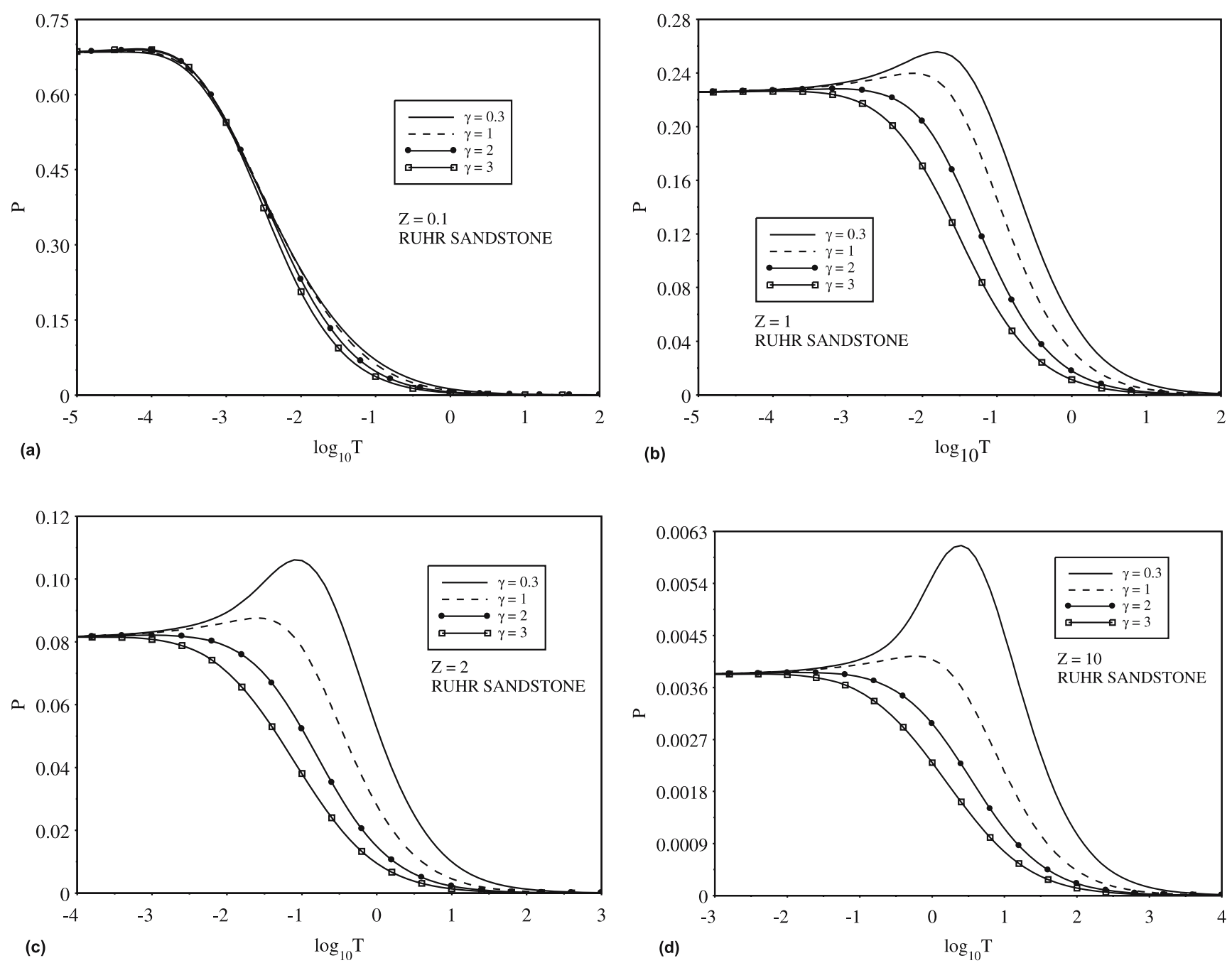

Figure 4. Influence of the value of the permeability anisotropy parameter $\gamma=\left(\chi_{r} / \chi_{z}\right)^{1 / 2}$ on the diffusion of the pore pressure $P$ with time $T$ for $r=0$ and (a) $Z=0.1$, (b) $Z=1$, (c) $Z=2$, (d) $Z=10$. The pore pressure rises above the initial undrained value before it decays to zero as $T \rightarrow \infty$ (Mandel-Cryer effect). This effect is more pronounced for smaller values of $\gamma$ and at greater depths.

This effect is more pronounced at greater depths and for smaller values of the permeability anisotropy parameter $\gamma=\left(\chi_{r} / \chi_{z}\right)^{1 / 2}$. Since, in general, $\chi_{r}>\chi_{z}$, the theoretical prediction of the Mandel-Cryer effect may get diluted in materials with anisotropic permeability. A similar conclusion was drawn in the two-dimensional plane strain analysis (Singh et al 2007).

Figure 5 shows the influence of the compressibility of the solid constituents of the poroelastic medium on the diffusion of the pore pressure with time. The dimensionless pore pressure $P$ at the point $r=0, z=2 a$ on the central line is computed as a function of the dimensionless time $T$ for four values of the Biot-Willis coefficient $\alpha$ for $\nu=0.25, \nu_{u}=0.27$ and $\gamma=0.1,1,10$. For a poroelastic medium with incompressible solid constituents, $\alpha=1$. We observe that the compressibility of the solid constituents of the poroelastic medium has a strong effect in the undrained limit $(T \rightarrow 0)$.

The effect of the compressibility of the fluid constituents of the poroelastic medium on the diffusion of the pore pressure with time is displayed in figure 6 . The dimensionless pore pressure $P$ at the point $r=0, z=2 a$ on the central line is computed as a function of the dimensionless time $T$ for four values of the undrained Poisson's ratio $\nu_{u}$ for $v=0.12, \alpha=0.65$ and $\gamma=0.1,1,10$. For a poroelastic medium with incompressible fluid constituents, $\nu_{u}=0.5$. We observe that the compressibility of the fluid constituents has a strong effect in the undrained state $(T \rightarrow 0)$. Figures 5 and 6 reveal that, in the short term, while the effect of the compressibility of the solid constituents is to increase the pore pressure, the effect of the compressibility of the fluid constituents is to decrease the pore pressure. 

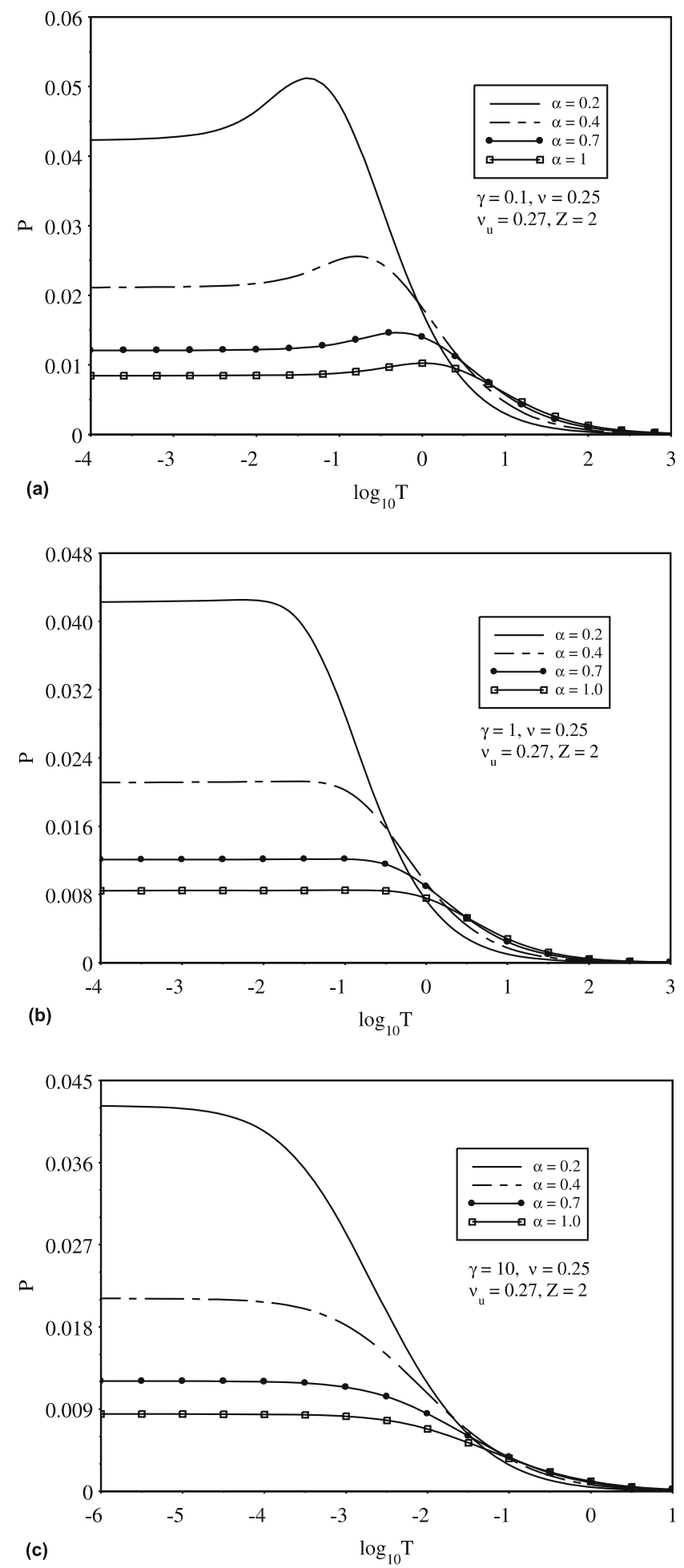

Figure 5. Effect of the value of the Biot-Willis coefficient $\alpha$ on the diffusion of the pore pressure $P$ with time $T$ for $Z=2, \nu=0.25, \nu_{u}=0.27$ and for (a) $\gamma=0.1$, (b) $\gamma=1.0$, (c) $\gamma=10$. For a poroelastic model with incompressible solid constituents, $\alpha=1$. The influence of the compressibility of the solid constituents is to increase the short-term pore pressure.

Figure 7 displays the depth profile of the pore pressure for Ruhr sandstone $\left(\nu=0.12, \nu_{u}=0.31\right.$, $\alpha=0.65)$ for three values of the dimensionless time
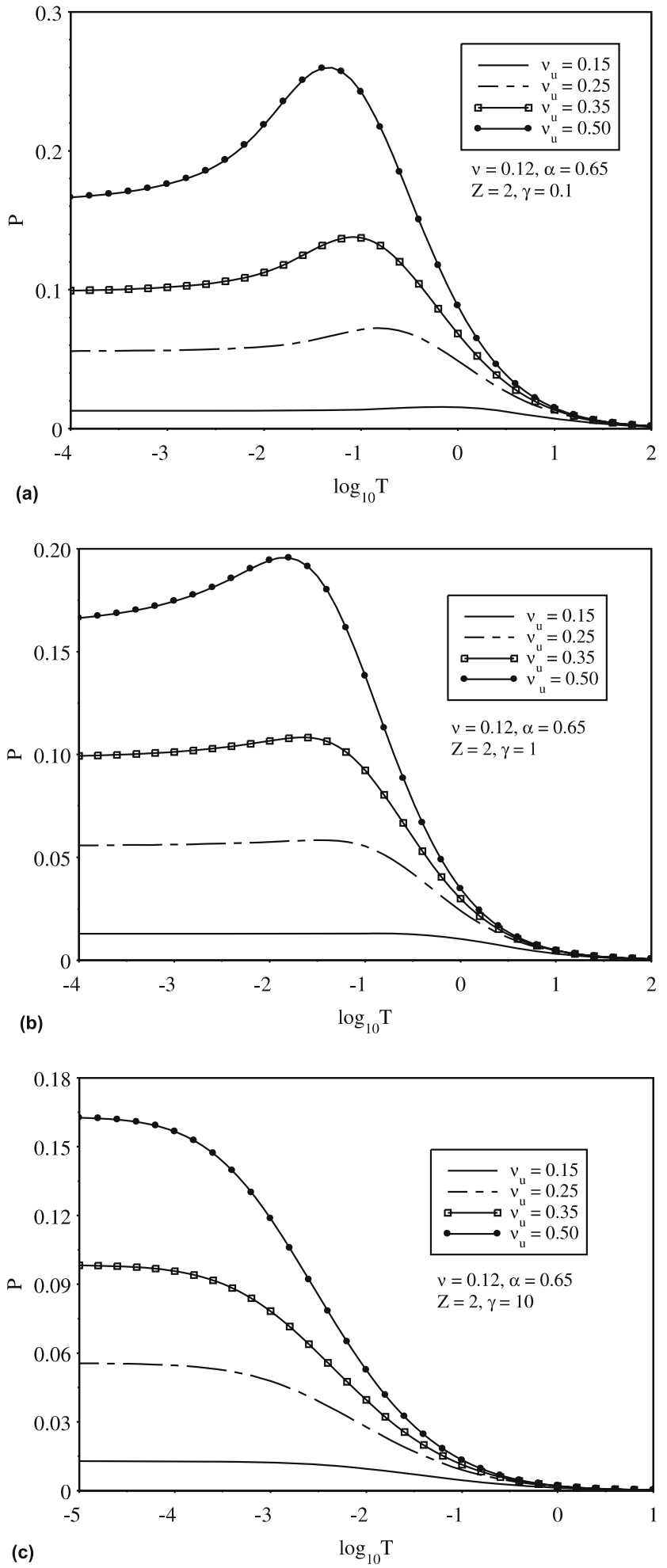

Figure 6. Effect of the value of the undrained Poisson's ratio $\nu_{u}$ on the diffusion of the pore pressure with time $T$ for $Z=2, \nu=0.12, \alpha=0.65$ and for (a) $\gamma=0.1$, (b) $\gamma=1.0$, (c) $\gamma=10$. For a poroelastic model with incompressible fluid constituents, $\nu_{u}=0.5$. The influence of the compressibility of the fluid constituents is to decrease the pore pressure.

$T$ for $\gamma=0.1,1,10$. Initially, a very large pore pressure develops near the surface resulting in a steep gradient of the pore pressure. 

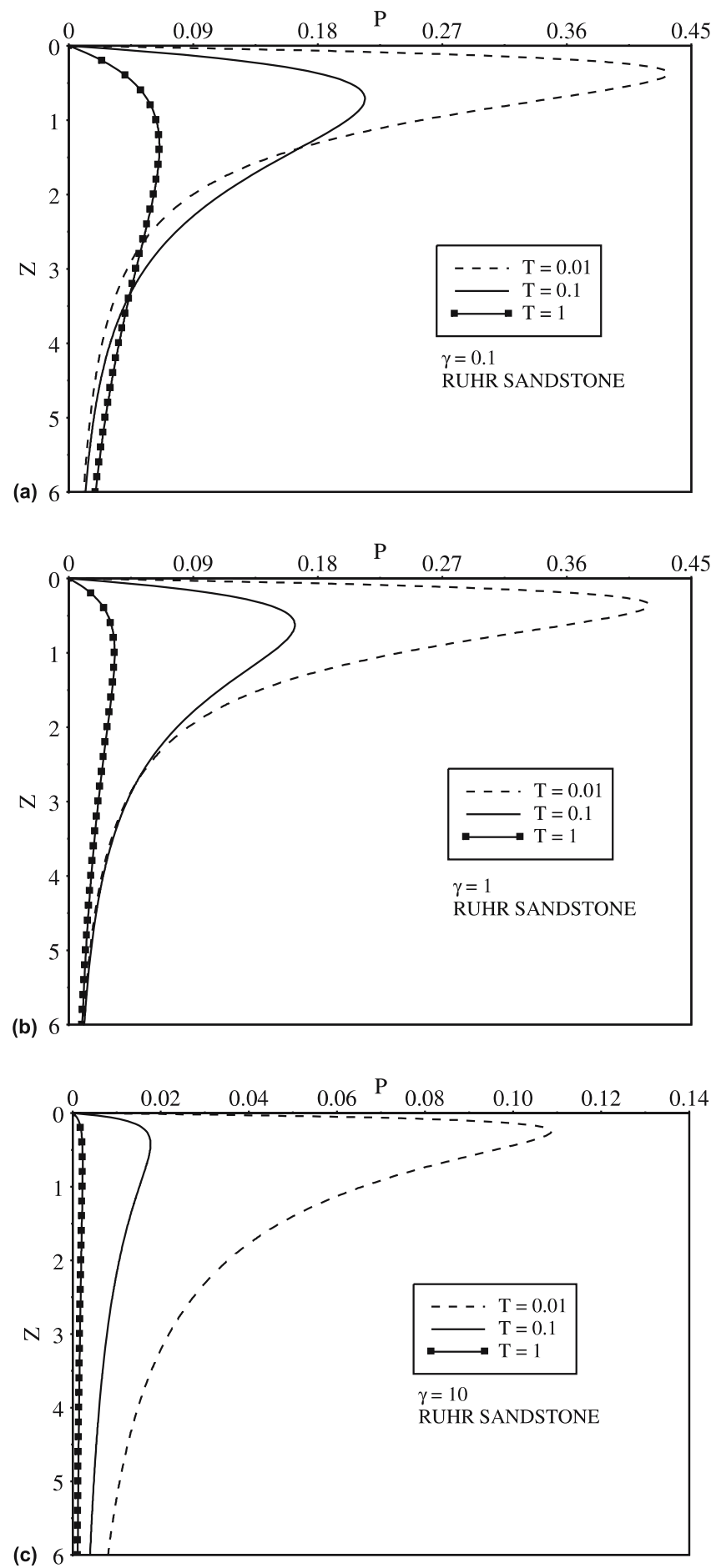

Figure 7. Depth profile of the dimensionless pore pressure $P$ at three time-instants for $r=0$ and (a) $\gamma=0.1$, (b) $\gamma=1.0$, (c) $\gamma=10$.

\section{Conclusions}

An analytical solution has been obtained of the governing equations representing the diffusiondeformation of a poroelastic medium possessing anisotropic permeability and compressible fluid and solid constituents. This solution has been used to study the quasi-static deformation of a halfspace by surface loading. As an example, the problem of the consolidation of a uniform half-space caused by normal disc loading has been discussed in detail. The effect of anisotropy in permeability and compressibilities of the fluid and solid constituents have been investigated. The following observations are made in relation to the consolidation of a poroelastic half-space by normal loads:

- The anisotropy in permeability may accelerate the consolidation process. However, it has no effect on the initial and the final settlements.

- The anisotropy in permeability may lead to a dilution in the theoretical prediction of the Mandel-Cryer effect.

- The compressibility of the solid constituents of the poroelastic medium may accelerate the consolidation process. However, it has no influence on the initial and final settlements.

- In the short term, the compressibility of the solid constituents increases the pore pressure.

- The compressibility of the fluid constituents increases the initial settlement. It has no influence on the final settlement.

- The compressibility of the fluid constituents decreases the short-term pore pressure.

The theory developed can be used for modelling the time-settlement of a poroelastic soil medium possessing anisotropic permeability and compressible fluid and solid constituents. This relates to an important class of problems in geotechnical engineering. The theory may also find applications in studies involving oil/gas reservoirs. Because such reservoirs are usually sandwiched between impermeable formations, the oil or water injected from the bore well or from the induced fracture flows mainly in the horizontal direction (Watanabe and Kurashige 1997). Therefore, the permeability in the horizontal direction will be much more than the permeability in the vertical direction.

\section{Acknowledgements}

The authors are thankful to the referees for their useful comments which led to improvements in the presentation of the paper. One of the authors (SJS) is thankful to the Indian National Science Academy for financial support under its Senior Scientist Scheme.

\section{References}

Biot M A 1941 General theory of three-dimensional consolidation; J. Appl. Phys. 12 155-164. 
Biot M A 1956 General solutions of the equations of elasticity and consolidation for a porous material; J. Appl. Mech. 78 91-98.

Booker J R and Carter J P 1986 Long term subsidence due to fluid extraction from a saturated, anisotropic, elastic soil mass; Q. J. Mech. Appl. Math. 39 85-98.

Booker J R and Carter J P 1987a Elastic consolidation around a point sink embedded in a half-space with anisotropic permeability; Int. J. Num. Anal. Meth. Geomech. 11 61-77.

Booker J R and Carter J P 1987b Withdrawal of a compressible pore fluid from a point sink in an isotropic elastic half space with anisotropic permeability; Int. J. Solids Struct. 23 369-385.

Booker J R and Randolph M F 1984 Consolidation of a cross-anisotropic soil medium; Q. J. Mech. Appl. Math. 37 155-164.

Chau K T 1996 Fluid point source and point forces in linear elastic diffusive half-spaces; Mech. Mater. 23 241-253.

Chen G J 2004 Consolidation of multilayered half space with anisotropic permeability and compressible constituents; Int. J. Solids Struct. 41 4567-4586.

Chen G J 2005 Steady-state solutions of multilayered and cross-anisotropic poroelastic half-space due to a point sink; Int. J. Geomech. 5 45-57.

Cryer C W 1963 A comparison of the three-dimensional consolidation theories of Biot and Terzaghi; Q. J. Mech. Appl. Math. 16 401-412.

Detournay E and Cheng A H D 1993 Fundamentals of poroelasticity; In: Comprehensive Rock Engineering: Principles, Practice and Projects (ed.) Hudson J A (Oxford: Pergamon Press) 2 113-171.
Ganbe T and Kurashige M 2001 Integral equations for a $3 \mathrm{D}$ crack in a fluid saturated poroelastic infinite space of transversely isotropic permeability; JSME Int. J. Ser A 44 423-430.

Pan E 1999 Green's functions in layered poroelastic half-spaces; Int. J. Numer. Anal. Meth. Geomech. 23 1631-1653.

Rajapakse R K N D and Senjuntichai T 1993 Fundamental solutions for a poroelastic half-space with compressible constituents; J. Appl. Mech. $60844-856$.

Rudnicki J W 1986 Fluid mass sources and point forces in linear elastic diffusive solids; Mech. Mater. 5 383-393.

Schapery R A 1962 Approximate methods of transform inversion for viscoelastic stress analysis; Proc. 4th US Nat. Congr. Appl. Mech. 2 1075-1085.

Senjuntichai T and Rajapakse R K N D 1995 Exact stiffness method for quasi-statics of a multilayered poroelastic medium; Int. J. Solids Struct. 32 1535-1553.

Singh S J and Rani S 2006 Plane strain deformation of a multilayered poroelastic half-space by surface loads; J. Earth Syst. Sci. 115 685-694.

Singh S J, Rani S and Kumar R 2007 Quasi-static deformation of a poroelastic half-space with anisotropic permeability by two-dimensional surface loads; Geophys. J. Int. 170 1311-1327.

Wang H F 2000 Theory of linear poroelasticity (Princeton: Princeton Univ. Press).

Wang R and Kuempel H-J 2003 Poroelasticity: Efficient modelling of strongly coupled, slow deformation processes in multilayered half-space; Geophysics 68 705-717.

Watanabe K and Kurashige M 1997 Fundamental solutions for fluid-saturated, isotropic, poroelastic, infinite solid having zero permeability in one direction; Appl. Mech. Engg. 2 53-71. 